Elisabeth França 1

José Moreira de Souza 2

Mark Drew Crosland Guimarães 1

Eugênio Marcos Andrade Goulart 3

Enrico Colosimo 4

Carlos Maurício de Figueiredo Antunes 5

\section{Associação entre fatores sócio-econômicos e mortalidade infantil por diarréia, pneumonia e desnutrição em região metropolitana do Sudeste do Brasil: um estudo caso-controle}

\author{
Association between socioeconomic factors \\ and infant deaths due to diarrhea, pneumonia, \\ and malnutrition in a metropolitan area \\ of Southeast Brazil: a case-control study
}

1 Grupo de Pesquisas em Epidemiologia, Departamento de Medicina Preventiva e Social, Faculdade de Medicina, Universidade Federal de Minas Gerais.

Av. Alfredo Balena 190, 9o andar, Belo Horizonte, MG 30130-100, Brasil.

efranca@medicina.ufmg.br

2 Fundação João Pinheiro.

Al. das Acácias 70

Belo Horizonte, MG

31275-150, Brasil.

3 Departamento de

Pediatria, Faculdade de

Medicina, Universidade

Federal de Minas Gerais.

Av. Alfredo Balena 190,

4o andar, Belo Horizonte, MG

30130-100, Brasil.

4 Departamento de

Estatística, Instituto

de Ciências Exatas,

Universi dade Federa

de Minas Gerais.

Av. Antônio Carlos 6627,

Belo Horizonte, MG

31270-901, Brasil.

5 Departamento de

Parasitologia, Instituto

de Ciências Biológicas,

Universidade Federal

de Minas Gerais.

Av. Antônio Carlos 6627,

Belo Horizonte, MG

31270-901, Brasil.
Abstract A population-based case control study was carried out to identify determinant factors for post-neonatal infant deaths due to diarrhea, pneumonia, and malnutrition in Greater Metropol itan Belo Horizonte, Southeast Brazil. From May 1, 1991, to April 30, 1992, 511 post-neonatal deaths due to diarrhea, pneumonia, and malnutrition were selected after investigation of medical records to vali date cause of death. Of this total, 396 deaths were compared to a neighborhood control group, matched for age. The study was carried out in a low-income area with a high proportion of families living in shantytowns. The article discusses the methodology and selected socioeconomic factors. Logistic regression analysis indicated that number of household appliances, mother's and father's education, and mother's marital and work status were significantly associated with risk of infant death, i.e., they were determinants of infant deaths due to avoidable causes.

Key words Infant Mortality; Socioeconomic Factors; Case-Control Studies

Resumo Para investigar fatores determinantes da mortalidadeinfantil pós-neonatal por diarréia, pneumonia e desnutrição, abordadas como um mesmo grupamento de causas, realizou-se um estudo caso-controle de base populacional na Região Metropolitana de Belo Horizonte. No período de maio de 1991 a abril de 1992, foram estudados 396 óbi tos após investi gação hospitalar da causa de óbito, os quais foram comparados a crianças residentes na mesma vizinhança, emparelhadas por idade. A pesquisa foi realizada em uma população de baixa renda, sendo que a mai oria das famílias entrevi stadas resi diam em favelas. Neste artigo se discutea metodol ogia utilizada e resultados rel evantes relativos aos fatores sócio-econômi cos associados às mortes infantis. Verificou-se que a existência de el etrodomésticos no domicílio, o nível de escolaridade materna e paterna, a si tuação conjugal da mãe e o fato desta trabal har fora permaneceram estatisticamente associados ao risco de óbito infantil, após ajuste pel as variáveis sóci o-econômicas consi deradas, uti lizando-se model o de regressão logística condi cional. Estes resultados evidenciam a importância do nível sócio-econômi co na determinação dos óbitos infantis por causas evitáveis.

Palavras-chave Mortalidade Infantil; Fatores Sócio-Econômicos; Estudos Caso-Controle 


\section{Introdução}

A mortalidade infantil no Brasil é ainda muito elevada. Mesmo a taxa de mortalidade infantil pós-neonatal, apesar de ser de mais fácil intervenção e apresentar uma tendência mais decrescente que a neonatal, era de 16,5 por 1.000 nascidos vivos na Região Metropolitana de Belo Horizonte (RMBH) em 1990, ainda excessivamente al ta se comparada a outros países da América Latina. Este alto índice decorre do peso relativo dos óbitos por diarréias, pneumonias e desnutrição, responsáveis pela maior parte das mortes infantis tardias em nosso meio (Szwarcwald et al., 1992). Estas patologias, por serem intrinsecamente relacionadas entre si na determinação do óbito infantil pós-neonatal (Carvalho et al., 1990; Mendonça et al., 1994; Saad, 1986) podem ser consideradas como um mesmo grupamento de causas de óbito, o complexo diarréia-pneumonia-desnutrição.

Diversos estudos têm demonstrado a importância dos fatores sócio-econômicos na determinação da saúde infantil. Entre estes, a educação da mãe e a renda têm sido considerados elementos básicos, por serem indicadores de disponibilidade de recursos e conhecimento ou comportamento em relação à saúde da criança (Caldwell, 1979; Pharoah \& Morris, 1979; Victora et al., 1992). Entretanto, ainda é pequena a discussão sobre o peso desses fatores na determinação das mortes infantis nos estratos sociais de baixa renda, onde sabidamente se concentram a maior parte dos óbitos por causas evitáveis e para onde devem ser direcionados os esforços de vigilância e controle da mortalidade infantil pós-neonatal. Ao mesmo tempo, existe ainda heterogeneidade em relação às variáveis utilizadas para a aferição dos diferenciais sócio-econômicos: alguns autores operacionalizam o conceito de classe social, outros utilizam ocupação, renda, educação, estado civil das mães e mesmo diversas variáveis relativas às condições de moradia (Bennett, 1992; Caldwell, 1979; Pharoah \& Morris, 1979; Victora et al., 1988). Essas diversas abordagens evidenciam a natureza multifatorial da mortalidade infantil e a importância de se mensurar mais de uma dimensão dos determinantes sociais (Durkin et al., 1994).

Para investigar fatores associados à mortalidade infantil pós-neonatal por diarréia-pneumonia-desnutrição na RM BH, delineou-se um estudo caso-controle. Foram coletadas variáveis relativas a quatro agrupamentos de determinantes: sócio-econômicos, ambientais e ligados à moradia da família, reprodutivos e de utilização de serviços de saúde. Neste artigo discutem-se os resultados relativos aos fatores sócio-econômicos, bem como a metodologia utilizada e as principais dificuldades encontradas em um estudo de base populacional, realizado em uma grande região metropolitana do Brasil.

\section{Materiais e métodos}

A área abrangida pelo estudo foi parte da RMBH, situada na Região Sudeste, Estado de Minas Gerais, constituída pela capital mineira, Belo Horizonte e mais 13 municípios (Betim, Caeté, Contagem, I birité, Lagoa Santa, Nova Lima, Pedro Leopoldo, Raposos, Ribei rão das Neves, Rio Acima, Sabará, Santa Luzia e Vespasiano) com uma população total de cerca de 3.360 .000 habitantes, segundo o Censo Demográfico de 1991 (IBGE, 1991).

O estudo constou de três etapas distintas: uma análise das Declarações de Óbito (DO), a revisão hospitalar das mesmas para definição da causa básica do óbito e seleção dos casos a serem investigados, e um estudo domiciliar dos óbitos selecionados e de controles vizinhos, para o levantamento das variáveis de exposição relevantes.

\section{Seleção dos casos e controles}

Foram definidos como casos todas as crianças que morreram com 28 dias de idade a menos de um ano, tendo como causa básica do óbito a diarréia, a pneumonia ou a desnutrição. Para a seleção dos mesmos, analisaram-se todas as DO relativas a óbitos pós-neonatais de crianças residentes na RMBH ocorridos de maio de 1991 a abril de 1992, coletadas de forma prospectiva por busca ativa semanal na Secretaria de Estado de Planejamento de Minas Gerais. Inicialmente, foi feita uma primeira seleção das DO com diarréia (códigos 001-009) segundo a 9a Revisão da Classificação Internacional de Doenças, Lesões e Causas de Óbito (CID-9) (OM S, 1985) ou pneumonia (códigos 480-486) ou desnutrição (códigos 260-269) citadas em qualquer campo da DO. Nesta fase, foram selecionadas outras causas básicas relacionadas, como anemias (códigos 280-281 e 285), desidratação (código 276), septicemia (código 038) e causas mal-definidas (códigos 779.9, 799.9, 785.5 e 786). Os óbitos por septicemia neonatal foram também analisados, com base em observações de estudo anterior, em que se detectou a utilização indevida pelos codificadores regionais do código 771-8 (infecções específicas do período perinatal) para os casos de septicemia 
pós-neonatal (CID 038) com conseqüente subregistro desta nas estatísticas oficiais publicadas no Estado a partir de 1984 (Mendonça et al., 1994).

Para a investigação da causa de óbito, um médico supervisor selecionava as DO para revisão do prontuário hospitalar, feita por pessoal treinado. Após o levantamento de dados relativos ao quadro clínico, resultados de exames e procedimentos importantes, a história clínica de cada caso individual era discutida com o responsável pela coleta de dados no hospital para a definição da causa básica do óbito. Posteriormente, era feita uma revisão por dois médicos pediatras, que avaliavam de maneira independente as informações coletadas e selecionavam os óbitos a serem incluídos na pesquisa, utilizando os seguintes critérios: a) óbito por diarréia: diarréia como quadro clínico predominante, antecedente às outras patologias associadas, sem desnutrição grave ou moderada associada; b) óbito por pneumonia: pneumonia como quadro clínico predominante, de preferência confirmada por radiografia de tórax, antecedente ou surgindo na mesma época de outras patologias associadas, sem desnutrição grave ou moderada associada; c) óbi to por desnutrição: desnutrição moderada ou grave, associada à diarréia e/ ou pneumonia. Considerou-se com desnutrição moderada ou grave toda criança com índice peso/idade igual ou inferior a menos dois escores $z$, ou seja, menos de dois desvios-padrão em relação ao peso esperado para a idade e sexo. A avaliação antropométrica foi realizada com o programa Epinut/Epi Info (Dean et al., 1994), que utiliza como padrão de referência a curva do NCHS National Center for Health Statistics - (Hamill et al., 1979) recomendado pela Organização Mundial da Saúde. Para as crianças com peso ao nascer inferior a $2.500 \mathrm{~g}$ e para aquelas com desidratação que levasse à internação, realizou-se análise individual para avaliar a possibilidade de falso positivo no diagnóstico de desnutrição moderada ou grave. No caso de crianças com DO inicialmente selecionadas, para as quais não se conseguiu localizar o prontuário hospitalar em pelo menos duas tentativas ou que tiveram história hospitalar não conclusiva, considerou-se a causa básica definida na DO e a história da doença relatada pela mãe na investigação domiciliar.

Para cada óbito foi selecionado um controle domiciliar vizinho ao caso, emparelhado por idade, sem história de doença grave nos últimos trinta dias. Para localizar o controle domiciliar, foram percorridas as casas próximas, sorteando a direção (esquerda ou direita). Em ca- so de domicílio vazio ou quando não era possível localizar a mãe da criança ou outro parente em duas outras tentativas, selecionava-se outro domicílio mais próximo, com criança nas condições especificadas.

\section{Coleta de dados relativos à exposição}

Na investigação domiciliar, foram coletadas informações com a mãe de cada caso e respectivo controle domiciliar, utilizando-se questionário e manual de instrução específicos. As variáveis ambientais foram obtidas através de observação. Realizou-se também um estudo piloto, com pré-teste dos instrumentos através de entrevistas com dez famílias. Após análise dos resultados, foram feitas adequações em algumas perguntas do questionário e decidiu-se incluir todos os óbitos, inclusive os com local de ocorrência não hospitalar, dada a sua possível magnitude (foram detectados quatro casos de óbito domiciliar no estudo piloto).

Teve-se especial cuidado na seleção dos estagiários responsáveis pelas entrevistas, sendo escolhidos somente entrevistadores do sexo feminino, por se supor que isto facilitaria o acesso e a colaboração das mães das crianças. O treinamento dos entrevistadores foi realizado durante uma semana e incluiu trabalho de campo com entrevistas e preenchimento de questionários. A local ização dos domicílios da amostra foi difícil, apesar de solicitação formal aos hospitais para preenchimento correto dos endereços nas DO, inclusive afixando cartazes (o preenchimento das variáveis de identificação do paciente na DO é geralmente feito por funcionários administrativos dos hospitais). A permissão para a realização da entrevista foi requerida formalmente através de uma carta de apresentação da entrevistadora à família da criança, solicitando consentimento da mãe para participação no estudo e assegurando a confidencialidade das informações coletadas. Na entrevista, foram coletadas variáveis maternas e relativas à criança, de utilização de serviços de saúde, além de variáveis relativas à moradia e ao nível sócio-econômico da família. A renda familiar do grupo de casos era a do mês de ocorrência do óbito e a do controle domiciliar era relativa ao mês anterior à entrevista. Para o levantamento da renda, que é sabidamente uma variável cuja coleta de informação é mais complexa e sujeita a viés de aferição, perguntou-se quanto cada pessoa recebeu no último mês, levando-se em conta todos os rendimentos. A codificação da renda familiar foi feita em relação ao valor do salário-mínimo vigente. As entrevistas realizadas nos meses de 
mudança de salário foram verificadas caso a caso na etapa de codificação. Além disso, valores discrepantes da renda individual informada foram conferidos com informações coletadas relativas à ocupação. A coleta de dados sobre eletrodomésticos referiu-se à existência no domicílio de rádio, televisão e geladeira.

Procurou-se garantir a qualidade das informações coletadas através de supervisão/ revisão semanal de todos os questionários. Realizou-se validação de uma amostra probabilística de $5 \%$ das entrevistas concluídas nos seis primeiros meses da pesquisa, com repetição de perguntas por entrevistador diferente. Os resultados foram considerados bons, sendo utilizados no monitoramento dos entrevistadores.

\section{Processamento e análise dos dados}

A primeira codificação dos dados coletados era feita pelo próprio entrevistador, no momento de revisão do questionário com a supervisão da investigação domiciliar, segundo um manual elaborado para a pesquisa. A codificação definitiva foi realizada quando todos os questionários já estavam prontos e foi conferida através de uma amostra probabilística sistemática dos questionários domiciliares.

Para a entrada de dados, optou-se pelo programa Foxpro (Fox Holdings, 1991) como gerenciador da base de dados. Para o processamento e análise, foram utilizados os programas Epi Info (Dean et al., 1994) e MULTLR (Campos Filho \& Franco, 1989). Um único profissional ficou responsável pela digitação dos dados. Para identificar eventuais erros, foi feita conferência visual, assim como avaliação de erros de amplitude e de consistência lógica.

$\mathrm{Na}$ análise estatística, as diferenças entre casos investigados e casos não localizados ou entre casos definidos por revisão de prontuário hospitalar e aqueles baseados na $\mathrm{DO} /$ investigação domiciliar, foram avaliadas pelo teste do qui-quadrado para amostras independentes. Para esta comparação, utilizaram-se as variáveis registradas na DO e, consequentemente, as categorizações aí padronizadas, inclusive a escolaridade materna. A classificação desta variável em três categorias ("nenhuma", "fundamental", "2o grau ou mais") apesar de se considerar pouco adequada, foi utilizada nessa comparação, pois seu objetivo foi somente analisar se a seleção da amostra não tinha sido diferencial.

A análise da associação entre o óbito infantil e os fatores de exposição considerados foi feita através da estimativa da odds relativa (OR) e intervalo de confiança a 95\%, obtida através do modelo de regressão logística condicional
(Hosmer \& Lemeshow, 1989). Os valores desconhecidos das variáveis, todos menores que $2 \%$, foram substituídos por aqueles da categoria mais freqüente, pois este critério de imputação não alterava as estimativas de risco, evitando assim perdas na análise dos dados. As associações envolvendo variáveis ordinais foram também testadas utilizando $\chi^{2}$ de tendência linear. O nível de significância de 0,05 foi adotado para os testes estatísticos realizados na análise.

\section{Resultados}

\section{Seleção da amostra}

Foram inicialmente analisadas 760 DO de óbitos pós-neonatais, sendo selecionadas 665 para investigação da causa de óbito (Figura 1). Nesta primeira etapa, foram excluídas as DO em que havia associação com malformação congênita importante e/ ou paralisia cerebral, outra infecção ou doença grave associada considerada como causa básica do óbito (como por exemplo, óbitos em que a desnutrição era citada associada à meningite bacteriana aguda) e causas perinatais, como septicemia em menores de dois meses de idade e prematuridade extrema associada (95 óbitos no total). Após revisão do prontuário hospitalar, foram excluídos outros 154 óbitos: associação com anomalia congênita importante ou paralisia cerebral ( $n=32$ ) definição de outra causa bási ca do óbito $(n=29)$ ou por esta ser inconclusiva $(n=47)$ além de outros motivos, como criança residente em município fora da área estudada $(n=12)$ e sel eção inicial incorreta $(n=5)$. Possíveis causas perinatais foram também excluídas ( $n=6$ ) após revisão cuidadosa de todas as crianças com relato de retenção em berçário ao nascimento superior a 15 dias. Foram também excluídos da investigação domiciliar os óbitos de crianças institucionalizadas ( $n=17$ ). Os óbitos de gêmeos foram analisados caso a caso, tendo sido incluídos, por sorteio, apenas um dos irmãos $(n=6)$.

Dos 511 óbitos selecionados nesta etapa, foi feita investigação domiciliar de 396 casos. Não se conseguiu encontrar as famílias de 115 crianças, após pelo menos duas tentativas infrutíferas de localização das mesmas. Isto ocorreu principalmente devido à mudança de endereço ( $25 \%$ dos casos) não localização do mesmo (30\%) ou o endereço ser de outra família (21\%). Em outros casos, menos freqüentes, não se conseguiu encontrar a mãe ou outro responsável no domicílio (10\%) ou esta recusou-se a participar do estudo (4\%) além de outros moti- 
vos (10\%). Estes óbitos foram considerados como perdas da investigação domiciliar ( $23 \%$ dos óbitos selecionados). Apesar dessas perdas terem reduzido o tamanho da amostra inicial, o número de crianças estudadas (396 casos e 396 controles) teve um poder de $90 \%$ para detectar uma OR de 1,7 ou maior, equivalente a $5 \%$ designificância estatística para testes bilaterais e assumindo que, em média, $25 \%$ dos controles estivessem expostos aos fatores de risco estudados.

Para verificar possível seletividade entre as perdas ocorridas, que poderia comprometer a validade das inferências, analisaram-se algumas variáveis. Quanto ao local de ocorrência do óbito, por exemplo, verificou-se que as perdas foram em sua maioria de óbitos hospitalares ( $n=67$ óbitos; $64,4 \%$ ) da mesma forma que os casos investigados ( $n=247 ; 64,5 \%$ ). Quanto à idade da criança ao óbito, idade materna e nível instrucional da mãe, as perdas também não mostraram diferenças significativas em relação aos casos investigados. Entretanto, em relação ao mês de ocorrência do óbito, verificou-se que houve uma maior proporção de perdas nos últimos meses de coleta de dados (Tabela 1).

Em relação ao critério de caso utilizado, 247 óbitos (62,4\% de 396) tinham informações hospitalares conclusivas, permitindo definir com maior segurança a causa básica do óbito. Para 149 mortes sem esta informação, considerouse a causa básica da DO (64,4\% destes após necrópsia) e/ ou após entrevista com os pais da criança (entrevista direcionada). Ao se comparar estes óbitos com os selecionados por história hospitalar conclusiva, verificou-se não ter havido diferença significativa $(p>0,05)$ para as variáveis idade da criança, idade e nível instrucional da mãe.

A entrevista domiciliar foi realizada cerca de dois meses após a ocorrência do óbito, com uma média de 1,6 visitas por entrevista completada (caso e controle, emparelhados por vizinhança e idade). A mãe da criança foi a informante em mais de $95 \%$ das entrevistas, tanto no grupo de casos quanto no de controles. Cerca de $6 \%$ dos óbitos tiveram a entrevista realizada seis ou mais meses após a sua ocorrência. Neste caso, foram consideradas as informações relativas à data da entrevista domiciliar.

\section{Comparação de casos e controles}

A maioria das famílias entrevistadas morava em favelas. A comparação entre casos e controles em relação ao local de moradia e idade da criança mostrou que o emparelhamento foi bem sucedido, com diferença não significativa entre casos e controles (Tabela 2).
Figura 1

Fluxograma do processo de seleção dos óbitos pós-neonatais investigados.

Região Metropolitana de Belo Horizonte, 1991-1992.

760 óbitos pós-neonatais com Diarréia, Pneumonia ou Desnutrição citadas na DO

1ạ etapa: Análise da DO
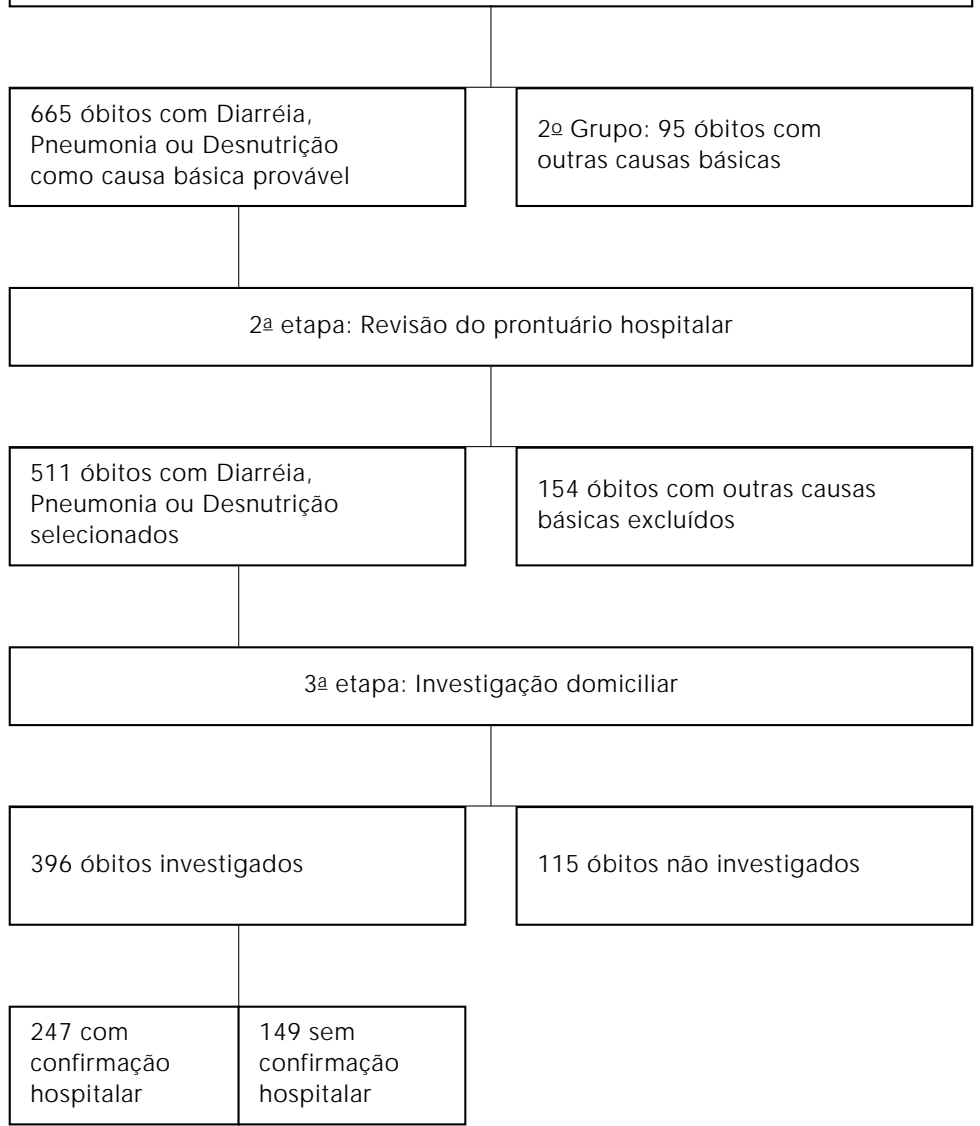

DO = Declaração de Ó bito.

A renda familiar média foi muito semelhante, sendo de 3,1 salários-mínimos (SM ) para os casos e 3,2 para os controles ( $p=0,67$ ) com $46 \%$ das famílias investigadas sobrevivendo com dois ou menos SM. Por outro lado, tanto a renda familiar per capita quanto a escolaridade da mãe e a do chefe da família estiveram significativamente associadas ao óbito infantil (Tabela 3). Para esta última variável, a categoria classificada como analfabeta (nenhuma escolaridade ou com curso rápido de alfabetização de adultos como MOBRAL - Movimento Brasileiro de Alfabetização) foi agrupada com pri- 
Tabela 1

Comparação de variáveis selecionadas da Declaração de Ó bito (DO) entre casos investigados e perdas. Região Metropolitana de Belo Horizonte, 1991-1992.

\begin{tabular}{|c|c|c|c|c|c|}
\hline \multirow[t]{2}{*}{ Variável } & \multicolumn{2}{|c|}{$\begin{array}{l}\text { Casos investigados } \\
\qquad(\mathrm{n}=396)\end{array}$} & \multicolumn{2}{|c|}{$\begin{array}{l}\text { Perdas } \\
(\mathrm{n}=115)\end{array}$} & \multirow[t]{2}{*}{$\begin{array}{l}\chi^{2} \\
(p)\end{array}$} \\
\hline & $n$ & $\%$ & $\mathrm{n}$ & $\%$ & \\
\hline \multicolumn{6}{|l|}{ Local de ocorrência do óbito* } \\
\hline Hospitalar & 247 & 64,5 & 67 & 64,4 & 0,0 \\
\hline Não hospitalar & 136 & 35,5 & 37 & 35,6 & $(0,99)$ \\
\hline \multicolumn{6}{|l|}{ Idade da criança (meses) } \\
\hline$<2$ & 45 & 11,4 & 8 & 7,0 & 9,1 \\
\hline $2-3$ & 116 & 29,3 & 33 & 28,7 & $(0,06)$ \\
\hline $4-5$ & 94 & 23,7 & 32 & 27,8 & \\
\hline $6-8$ & 95 & 24,0 & 19 & 16,5 & \\
\hline $9-11^{* *}$ & 46 & 11,6 & 23 & 20,0 & \\
\hline \multicolumn{6}{|l|}{ Idade da mãe (anos) } \\
\hline$<20$ & 60 & 15,1 & 24 & 20,9 & 2,7 \\
\hline $20-34$ & 223 & 56,3 & 58 & 50,4 & $(0,43)$ \\
\hline $35 \mathrm{e}+$ & 28 & 7,1 & 10 & 8,7 & \\
\hline Ignorado & 85 & 21,5 & 23 & 20,0 & \\
\hline \multicolumn{6}{|l|}{ Escolaridade da mãe } \\
\hline Nenhuma & 36 & 9,1 & 10 & 8,7 & 0,24 \\
\hline Fundamental & 250 & 63,1 & 74 & 64,4 & $(0,97)$ \\
\hline 2 o grau ou + & 5 & 1,3 & 2 & 1,7 & \\
\hline Ignorado & 105 & 26,5 & 29 & 25,2 & \\
\hline \multicolumn{6}{|l|}{ Mês do óbito } \\
\hline Maio-J unho/1991 & 99 & 25,0 & 10 & 8,7 & 24,9 \\
\hline J ulho-Agosto/1991 & 66 & 16,6 & 13 & 11,3 & $(0,00)$ \\
\hline Setembro-O utubro/1991 & 51 & 12,9 & 11 & 9,6 & \\
\hline Novembro-Dezembro/1991 & 51 & 12,9 & 20 & 17,4 & \\
\hline J aneiro-Fevereiro/1992 & 72 & 18,2 & 34 & 29,5 & \\
\hline Março-Abril/1992 & 57 & 14,4 & 27 & 23,5 & \\
\hline
\end{tabular}

mário incompleto (pessoas que cursaram alguma série do primário ou foram alfabetizadas tardiamente) por não ter havido diferença significativa no intervalo de confiança da OR dessas categorias em relação à categoria referência (oito ou mais anos de estudo).

O número de eletrodomésticos na moradia mostrou-se significativamente associado ao risco de óbito infantil, com OR de 3,38 (IC a $95 \%$ de $2,11-5,41$ ) para as famílias que não tinham nenhum eletrodoméstico em relação àquelas com rádio, televisão e geladeira. A presença de somente um ou dois eletrodomésticos no domicílio, geralmente a posse de rádio e/ ou tel evisão, também representou um fator de risco significativo para o óbito.
Em relação à situação conjugal das mães, verificou-se que quase metade delas nos controles eram casadas, com proporção semelhante à de mães em união consensual ou sozinhas entre os casos. Estas duas últimas categorias (em união consensual e sozinhas) foram agrupadas em uma única (não casadas), porque a comparação destas categorias com a das mães casadas não mostrou diferença significativa entre elas. O risco de óbito infantil mostrou-se estatisticamente associado ao fato de a mãe relatar não ser casada, com OR de 2,47 (IC a 95\% de 1,82-3,35). Da mesma forma, o trabalho da mãe fora de casa foi estatisticamente significativo.

Após a inclusão de todas as variáveis sócioeconômicas no modelo de regressão logístico, o número de eletrodomésticos, o nível de escolaridade dos pais, a situação conjugal da mãe e o fato desta trabalhar fora permaneceram significativamente associados ao maior risco de óbito infantil.

\section{Discussão}

No presente estudo, foram selecionados para investigação 511 óbitos pós-neonatais por diarréia, pneumonia ou desnutrição, o que representou cerca de $90 \%$ das mortes registradas para a população infantil da região. Como o subregistro de mortes na RMBH é pouco importante (Viegas \& Dolabela, 1986) as declarações de óbito podem ser consideradas uma fonte confiável em termos quantitativos. Entretanto, devido à pequena confiabilidade da declaração de causa básica na DO (Carvalho et al., 1990; Mendonça et al., 1994), procurou-se utilizar um critério de caso mais sensível no início, coletando-se todas as DO com citação de diarréia, pneumonia ou desnutrição e outras causas relacionadas ou mal-definidas.

Os casos selecionados pela DO deveriam ter sido confirmados por investigação hospitalar, pois acreditava-se que mais de $90 \%$ deles tiveram atendimento hospitalar antes do óbito. Entretanto, com a realização do estudo piloto, verificou-se que mais de um terço das mortes eram não hospitalares, proporção semel hante à detectada na Região Metropolitana do Rio de Janeiro em 1986 (Niobey et al., 1992). Estes óbitos, se não incluídos, poderiam introduzir viés de sel eção no estudo. Optou-se então por um critério de caso menos específico, sendo considerada a causa básica definida na DO e/ ou no relato da mãe. Verificou-se que os casos selecionados por este critério não diferiram significativamente daqueles sel ecionados por revisão do prontuário hospitalar. 
Para a escolha dos controles populacionais, utilizou-se como critério de seleção crianças vizinhas aos casos com idades próximas. Os critérios de exclusão aplicados aos controles foram semelhantes aos casos, evitando-se a seleção de crianças com doenças graves, como anomalias congênitas importantes e outras. Apesar da opção pelo controle de vizinhança, feita principalmente por razões logísticas, verificou-se diferença entre casos e controles em relação a resi dir em bairros, favelas ou conjunto habitacional. Isto provavelmente ocorreu devido ao pareamento individual por idade, com dificuldade de localização de crianças com idade semelhante na vizinhança mais próxima do caso, obrigando o entrevistador a percorrer muitas vezes um grande número de moradias.

Para minimizar possíveis vícios de informação no estudo, pelo não mascaramento do entrevistador quanto à situação de ser caso ou controle, foi feita uma fiscalização rígida dos procedimentos de entrevista, procurando-se evitar que os entrevistadores tivessem conhecimento das hipóteses do estudo. O viés de memória foi também atenuado, pois se conseguiu entrevistar as famílias dos casos em data próxima da época da ocorrência do óbito (semeIhante, portanto, à da pesquisa dos controles) e a mãe da criança foi a informante na maioria das entrevistas de casos e controles.

A proporção de $23 \%$ de perdas de casos e controles foi relativamente alta, apesar da prioridade dada à local ização dos endereços, considerada um aspecto crítico em estudos realizados em grandes regiões metropolitanas do Brasil, com índices de perdas geralmente altos (Formigli et al., 1996; Niobey et al., 1992). No estudo, verificou-se que as perdas não foram diferenciais para as variáveis idade e educação da mãe, que são relacionadas com o nível sócio-econômico da família, sugerindo não ter havido comprometimento da representatividade da amostra. Neste aspecto, a maior diferença detectada entre as perdas e a amostra investigada foi em relação ao mês de ocorrência do óbito, de menor importância, pois não foi objeto do presente estudo uma análise da distribuição sazonal da mortalidade infantil. Podese supor que as perdas ocorridas, motivadas principalmente pela não localização dos endereços, se distribuídas heterogeneamente entre expostos e não expostos, tenham levado a uma diminuição das medidas de risco. As estimativas de OR encontradas foram, entretanto, muito significativas, o que nos leva a considerar que a perda de casos não comprometeu as inferências feitas no estudo.

\begin{tabular}{|c|c|c|c|c|c|}
\hline \multirow[t]{2}{*}{ Característica } & \multicolumn{2}{|c|}{ Casos } & \multicolumn{2}{|c|}{ Controles } & \multirow{2}{*}{$\begin{array}{l}\chi^{2} \\
(p)\end{array}$} \\
\hline & $\mathrm{n}$ & $\%$ & $\mathrm{n}$ & $\%$ & \\
\hline \multicolumn{6}{|l|}{ Local de moradia* } \\
\hline Bairro & 159 & 40,9 & 168 & 43,2 & 2,85 \\
\hline Conjunto habitacional & 18 & 4,6 & 27 & 6,9 & $(0,24)$ \\
\hline Favela & 212 & 54,5 & 194 & 49,9 & \\
\hline \multicolumn{6}{|l|}{ Idade da criança (meses) } \\
\hline$<2$ & 45 & 11,4 & 47 & 11,9 & 1,04 \\
\hline $2-3$ & 114 & 28,8 & 107 & 27,0 & $(0,90)$ \\
\hline $4-5$ & 98 & 24,7 & 91 & 23,0 & \\
\hline $6-8$ & 90 & 22,7 & 99 & 25,0 & \\
\hline $9-11$ & 49 & 12,4 & 52 & 13,1 & \\
\hline
\end{tabular}

* sete pares de zona rural excluídos.

A pesquisa foi realizada em uma população de baixo nível sócio-econômico, com casos e controles semel hantes em relação à renda familiar (média de três salários-mínimos). É provável que esta similaridade da renda tenha ocorrido devido à opção por controles de vizinhança, com possível superpareamento em relação às variáveis sócio-econômicas. A renda familiar per capita, entretanto, foi significativamente menor entre os casos, devido ao maior tamanho da família entre estes. Segundo McCracken (1990), o recurso disponível familiar é afetado pelo número de pessoas, principalmente em famílias de baixa renda. Na análise multivaria$d a$ a renda diminuiu seu efeito com a inclusão de outras variáveis sócio-econômicas correlacionadas, como o número de eletrodomésticos na casa. Esta última variável, freqüentemente utilizada em pesquisas de opinião pública para classificação sócio-econômica, manteve-se significativa após o ajuste, mostrando-se um bom preditor do risco de óbito infantil, com associação estatisticamente significativa também no teste de qui-quadrado de tendência. Desta forma, tanto ter um ou dois eletrodomésticos (geralmente rádio e televisão) quanto não ter nenhum, representaram fatores de risco, quando comparados à categoria referência (ter três eletrodomésticos). Este achado é semelhante ao relatado por Olinto et al. (1993), em um estudo sobre determinantes de desnutrição infantil em população de baixa renda de Pelotas, no qual o número de utilitários domésticos foi a única variável que manteve seu efeito sobre o déficit de peso-altura no modelo com outras 
Distribuição de casos e controles de acordo com variáveis sócio-econômicas selecionadas. Região Metropolitana de Belo Horizonte, 1991-1992.

\begin{tabular}{|c|c|c|c|c|}
\hline Característica & Casos & Controles & OR (IC 95\%) & OR ajustada* (IC 95\%) \\
\hline \multicolumn{5}{|c|}{ Renda per capita (salários mínimos)** } \\
\hline $0,00-0,24$ & 100 & 62 & $2,75(1,63-4,63)$ & $1,38(0,75-2,55)$ \\
\hline $0,25-0,49$ & 123 & 124 & $1,59(0,99-2,56)$ & $1,04(0,60-1,79)$ \\
\hline $0,50-0,99$ & 122 & 134 & $1,37(0,87-2,17)$ & $1,01(0,60-1,72)$ \\
\hline$\geq 1,00$ & 51 & 76 & 1,00 & 1,00 \\
\hline$\chi^{2}(3 g \mid)$ & & & $16,68(p=0,001)$ & \\
\hline \multicolumn{5}{|l|}{ Eletrodomésticos em casa** } \\
\hline Nenhum & 88 & 50 & $3,38(2,11-5,41)$ & $2,01(1,17-3,45)$ \\
\hline 1-2 diferentes & 202 & 173 & $2,11(1,50-2,97)$ & $1,45(0,98-2,13)$ \\
\hline Pelo menos 3 diferentes & 106 & 173 & 1,00 & 1,00 \\
\hline$\chi^{2}(2 g \mid)$ & & & $32,78(p=0,000)$ & \\
\hline \multicolumn{5}{|l|}{ Escolaridade da mãe** } \\
\hline Até 3 anos & 195 & 136 & $5,14(2,74-9,64)$ & $2,68(1,31-5,50)$ \\
\hline 4-7 anos & 178 & 199 & $2,95(1,61-5,41)$ & $2,00(1,02-3,89)$ \\
\hline$\geq 8$ anos & 23 & 61 & 1,00 & 1,00 \\
\hline$\chi^{2}(2 g l)$ & & & $34,74(p=0,000)$ & \\
\hline \multicolumn{5}{|c|}{ Escolaridade do chefe de família** } \\
\hline Até 3 anos & 202 & 144 & $3,52(2,08-5,97)$ & $1,86(1,02-3,38)$ \\
\hline 4-7 anos & 165 & 187 & $2,07(1,24-3,45)$ & $1,38(0,78-2,43)$ \\
\hline$\geq 8$ anos & 29 & 65 & 1,00 & 1,00 \\
\hline$\chi^{2}(2 g l)$ & & & $27,47(p=0,000)$ & \\
\hline \multicolumn{5}{|l|}{ Situação conjugal da mãe } \\
\hline Sozinha/União consensual & 286 & 201 & $2,47(1,82-3,35)$ & $1,81(1,30-2,53)$ \\
\hline Casada & 110 & 195 & 1,00 & 1,00 \\
\hline$\chi^{2}(1 \mathrm{gl})$ & & & $37,10(p=0,000)$ & \\
\hline \multicolumn{5}{|l|}{ Trabalho da mãe fora de casa } \\
\hline Sim & 84 & 56 & $1,70(1,15-2,51)$ & $1,71(1,10-2,68)$ \\
\hline Não & 312 & 340 & 1,00 & 1,00 \\
\hline$\chi^{2}(1 \mathrm{gl})$ & & & $7,34(p=0,007)$ & \\
\hline
\end{tabular}

Nota: Utilizou-se o método de máxima verossimilhança condicional no cálculo da OR.

* ajustada para as demais variáveis apresentadas.

** $p<0,05$ no teste de $\chi^{2}$ de tendência linear.

variáveis sócio-econômicas. Neste estudo, a presença de três eletrodomésticos no domicílio incluía a posse de geladeira, além de rádio e televisão. É provável, portanto, que a variável eletrodomésticos no domicílio incorpore também a questão dos cuidados preventivos e medidas higiênicas, devido à maior facilidade para a conservação de alimentos. Ao mesmo tempo, ao mensurar o poder aquisitivo e o acesso a bens de consumo, esta variável representa uma medida objetiva de renda empregada em determinada direção, no sentido de poupança e garantia de condições efetivas/concretas de subsistência, portanto menos sujeita a variações no tempo, como ocorre com a renda per capita.

A escolaridade do chefe da casa, considerada importante determinante da situação social da família (Monteiro, 1988) também diminuiu seu efeito após ajustamento pelas outras variáveis sócio-econômicas consideradas, por sua provável colinearidade com a escolaridade da mãe (inclusive cerca de $10 \%$ das famílias pesquisadas tinham a mãe como chefe da casa).

Há mais de 15 anos já se considerava a educação materna como um importante determi- 
nante da mortalidade infantil. Mães com meIhor nível educacional teriam mais conhecimento e dariam maior importância à limpeza da moradia e hábitos higiênicos relativos à criança, reduzindo o nível de exposição aos patógenos (Caldwell, 1979). No presente estudo, essa variável manteve-se significativa, mesmo após inclusão no modelo das variáveis renda, escolaridade do chefe da família e existência de bens de consumo duráveis na moradia. Dessa forma, a educação materna teria um efeito próprio, independente de outros fatores sócioeconômicos, conforme verificado em estudo de coorte no sul do país (Victora et al., 1992).

As crianças nascidas de mães casadas tiveram maiores chances de sobrevivência do que as de famílias com mães em união consensual ou sozinhas. O estado civil é um indicador da posição da mulher dentro da família e, segundo McCracken (1990), o maior poder de decisão da mãe na unidade familiar é importante para a sobrevivência da criança.

Das famílias pesquisadas, $21 \%$ dos casos e $14 \%$ dos controles informaram que a mãe trabal hava fora. As ocupações relatadas eram de baixa remuneração: empregada doméstica (36\% das mães), faxineira (18\%), passadeira (8\%), cozinheira/ doceira/ balconista de bar (4\%). Interessante observar que o número de mães que utilizavam creches foi muito pequeno (6\%). A maioria informou deixar as crianças por conta de parentes adultos ( $59 \%$ dos casos) ou mesmo com outras crianças, quando saía para trabaIhar. O risco de morte infantil associado ao trabalho da mãe fora de casa talvez esteja ligado às maiores dificuldades de cuidado destas crianças, inclusive com suspensão mais precoce do aleitamento materno, conforme sugerido por Hobcraft et al . (1984). Entretanto, é possível que a OR observada neste estudo possa estar superestimada devido à conduta de se priorizar a entrevista com a mãe e, só em casos extremos, outros membros da família. Desta forma, a seleção dos controles pode ter levado em conta o fato de a mãe ou outro parente próximo estar presente em casa no momento da entrevista. A presença da mãe seria mais provável no caso das que não trabalhavam fora. Outras exposições relacionadas com este fato, como o nível de escolaridade materno, também podem ter sido afetadas, neste caso com OR subestimada (é provável que as mães dos controles que trabalhavam fora tivessem melhor nível de escolaridade). Acresce-se a isto a pouca confiabilidade desta informação, pois um maior número de mães informaram positivamente o quesito, quando indagadas se trabalhavam fora durante a gestação da criança (31\% dos ca- sos e 33\% dos controles). Essa diferença na informação obtida em relação ao trabalho materno de acordo com a época (por ocasião da gestação ou no momento da entrevista/ data do óbito) pode, entretanto, ser devida à alta rotatividade entre trabalho remunerado e domiciliar, freqüente entre mulheres de baixa renda (Facchini, 1995). Essas limitações, ainda pouco discutidas na literatura, indicam a necessidade de melhor explorar essa variável em outras investigações.

Em síntese, apesar das dificuldades encontradas, relacionadas principalmente à necessidade de investigação hospitalar para a determinação da causa de morte e a difícil localização das famílias para a entrevista, além das limitações metodológicas acima discutidas, este estudo demonstrou a utilidade do delineamento caso-controle na abordagem de determinantes da mortalidade infantil por causas evitáveis. Não se pretendeu aqui esgotar essa discussão, mas somente apresentar alguns resultados relativos aos fatores sócio-econômicos, que, em um modelo teórico de determinação, situam-se em uma posição hierárquica superior aos demais determinantes (Olinto et al., 1993). Estes fatores, apesar da complexidade de sua abordagem para a intervenção, representam um enfoque central para os epidemiologistas no desvelamento das desigualdades em saúde e na procura de estratégias para sua superação (Goldbaum, 1997). No estudo foi possível evidenciar a existência de diferenças sociais em saúde, utilizando-se algumas medidas objetivas, como os anos de escolaridade da mãe e o número de eletrodomésticos no domicílio. Os resultados encontrados reforçam a importância dos fatores sócio-econômicos na ocorrência das mortes infantis evitáveis, mesmo em subgrupos populacionais de baixa renda e, ao mesmo tempo, indicam certa autonomia e poder discriminativo dos mesmos, chamando a atenção para os variados mecanismos através dos quais opera a desigualdade social na determinação da saúde infantil. 


\section{Agradecimentos}

Aos professores Maria do Carmo Leal e Paulo Chagastelles Sabroza, coordenadores da pesquisa Determinantes da Mortalidade Infantil na Região Metropolitana do Rio de Janeiro - 1986/1987, pelo incentivo e apoio metodológico no delineamento desta pesquisa, e a todos os bolsistas que foram fundamentais na realização do trabal ho de campo e no preparo dos dados para a análise. Esta pesquisa recebeu apoio financeiro da Organização Pan-Americana de Saúde (OPAS) e da Fundação de Amparo à Pesquisa do Estado de Minas Gerais (FAPEMIG).

\section{Referências}

BENNETT, T., 1992. Marital status and infant health outcomes. Social Science and Medicine, 35:11791187.

CALDWELL, J. C., 1979. Education as a factor in mortality decline: An examination of Nigerian data. Population Studies, 33:395-413.

CAM POS FILHO, N. \& FRANCO, E. L., 1989. A microcomputer program for multiple logistic regression by unconditional and conditional maximum likelihood methods. American Journal of Epidemiology, 129:439-444.

CARVALHO, M. L.; NIOBEY, F. M.; MIRANDA, N. N. \& SABROZA, P. C., 1990. Concordância na determinação da causa básica de óbito em menores de um ano de idade na Região Metropolitana do Rio de Janeiro, 1986. Revista de Saúde Pública, 24:2027.

DEAN, A. G.; DEAN, J. A.; COULOMBIER, D.; BRENDEL, K. A.; SMITH, D. C.; BURTON, A. H.; DICKER, R. C.; SULLIVAN, K.; FAGAN, R. F. \& ARNER, T. G., 1994. Epi Info, Version 6: A Word Processing, Databaseand Statistics Program for Epidemiology on Microcomputers. Atlanta: Centers of Disease Control and Prevention.

DURKIN, M. S.; ISLAM, S.; HASAN, Z. M. \& ZAMAN, S. S., 1994. Measures of socioeconomic status for child health research: Comparative results from Bangladesh and Pakistan. Social Science and Medicine, 38:1289-1297.

FACHINI, L. A., 1995. Trabalho Materno e Ganho de Peso Infantil. Pelotas: Editora Universitária.

FORMIGLI, V. L. A.; VIEIRA-DA-SILVA, L. M.; CERDEIRA, A. J. P.; PINTO, C. M. F.; OLIVEIRA, R. S. A.; CALDAS, A. C.; VILAS-BOAS, M. J. B.; FONSECA, A. C.; SOUZA, L. S. F.; SILVA, L. R. \& PAES, M. S. F., 1996. Avaliação da atenção à saúde através da investigação de óbitos infantis. Cadernos de Saúde Pública, 12(Sup. 2):33-41.
FOX HOLDINGS, 1991. Foxpro. Perrysburg: Fox Holdings.

GOLDBAUM, M., 1997. A epidemiologia em busca da eqüidade em saúde. In: EqüidadeeSaúde: Contribuições da Epidemi ologia (R. B. Barata, M. L. Barreto, N. Almeida Filho \& R. P. Veras, org.), pp. 6380, Rio de Janeiro: Editora Fiocruz/ ABRASCO.

HAMILL, P. V. V.; DRIZD, T. A.; JOHNSON, C. L.; REED, R. B.; ROCHE, A. F. \& MOORE, W. M., 1979. Physical growth: National Center for Health Statistics percentiles. American Journal Clinical Nutrition, 32:607-629.

HOBCRAFT, J. N.; MCDONALD, J. W. \& RUTSTEIN, S. O., 1984. Socio-economic factors in infant and child mortality: A cross-national comparison. Population Studies, 38:193-223.

HOSMER, D. W. \& LEMESHOW, S., 1989. Applied Logistic Regression. New York: John Wiley \& Sons.

IBGE (Fundação Instituto Brasileiro de Geografia e Estatística), 1991. Censo Demográfico; Resultados do Universo, Características da População e dos Domicílios. Rio de Janeiro: IBGE.

MCCRACKEN, S. D., 1990. A Multi-Level Socio-Demographic Analysis of Early Childhood Mortality in Brazil. Ph.D. Thesis, Austin: Texas University.

MENDONÇA, E. F.; GOULART, E. M. A. \& MACHADO, J. A. D., 1994. Confiabilidade da declaração de causa básica de mortes infantis em região metropolitana do sudeste do Brasil. Revista de Saúde Pública, 28:385-391.

MONTEIRO, C. A., 1988. Saúde e Nutrição das Crianças de São Paulo. São Paulo: Editora Hucitec.

NIOBEY, F. M. L.; DUCHIADE, M. P.; VASCONCELOS, A. G. G.; CARVALHO, M. L.; LEAL, M. C. \&VALENTE, J. G., 1992. Fatores de risco para morte por pneumonia em menores de um ano em uma região metropolitana do sudeste do Brasil. Um estudo casocontrole. Revista deSaúdePública, 26:229-238. 
OLINTO, M. T. A.; VICTORA, C. G.; BARROS, F. C. \& TOMASI, E., 1993. Determinantes da desnutrição infantil em uma população de baixa renda: Um modelo de análise hierarquizado. Cadernos de SaúdePública, 9(Sup.):14-27.

OMS (Organização Mundial de Saúde), 1985. Manual da Classificação Estatística Internacional deDoenças, Lesões e Causas de Óbitos - Nona Conferência de Revisão. São Paulo: Centro Brasileiro de Classificação de Doenças em Português.

PHAROAH, P. O. D. \& MORRIS, J. N., 1979. Postneonatal mortality. Epidemiology Reviews, 1:170-183.

SAAD, P. M., 1986. Mortalidade infantil por causas no estado de São Paulo (Brasil) em 1983. Análise sob a perspectiva das causas múltiplas de morte. Revista de Saúde Pública, 20:481-488.

SZWARCWALD, C. L.; CHERQUER, P. \& CASTILHO, E. A., 1992. Tendências da mortalidade infantil no Brasil nos anos 80. Informe Epidemiológico do SUS, 1:35-50.
VICTORA, C. G.; HUTTLY, S. R. A.; BARROS, F. C.; LOMBARDI, C. \& VAUGHAN, J. P., 1992. Maternal education in relation to early and late child health outcomes: Findings from a Brazilian cohort study. Social Science and Medicine, 34:899-905.

VICTORA, C. G.; SMITH, P. G.; VAUGHAN, J. P.; NOBRE, L. C.; LOMBARDI, C.; TEIXEIRA, A. M. B.; FUCHS, F. C.; MOREIRA, L. B.; GIGANTE, L. P. \& BARROS, F. C., 1988. Water supply, sanitation and housing in relation to the risk of infant mortality from diarrhoea. International Journal of Epidemiology, 17:651-654.

VIEGAS, J. M. S. \& DOLABELA, R. F., 1986. Estudo qualitativo das estatísticas de nascimentos e óbitos; O sub-registro em Minas Gerais e Região Metropolitana de Belo Horizonte - Resultados definitivos do Censo Demográfico de 1980. Indicadores deConjuntura, 8:485-495. 